\title{
Caracterização clínico-nutricional de japoneses e descendentes nipo-brasileiros na cidade de Campo Grande, MS, Brasil
}

\section{Clinical and nutricional characterization of japanese and japanese-brazilian descendants in Campo Grande city, MS, Brazil}

Caracterización nutricional clínica de los descendientes de japoneses y japoneses en la ciudad de Campo Grande, MS, Brasil

Lígia Aurélio Bezerra Maranhão Mendonça ${ }^{1}$ Rosângela dos Santos Ferreira ${ }^{2}$ Jackeline Satie Minami ${ }^{3}$

${ }^{1}$ Graduada em Nutrição pela Universidade Católica Dom Bosco (UCDB).

Especialista em Didática e Metodologia do Ensino Superior pelo Centro Universitário Anhanguera. Mestre em Biotecnologia UCDB. Pós-Graduanda em Nutrição Clínica Funcional pela Universidade Cruzeiro do Sul e Doutoranda em Biotecnologia pela Universidade Católica Dom Bosco. E-mail: Imendoncanutri@gmail.com

2 Pós-doutoranda em Biotecnologia pela Universidade Católica Dom Bosco(UCDB). Graduada em Nutrição pela Universidade Santa Úrsula. Mestrado e doutora em Saúde e Desenvolvimento na Região CentroOeste pela Universidade Federal de Mato Grosso do Sul (UFMS). Nutricionista do Hospital Universitário Maria Aparecida Pedrossian da UFMS. E-mail: rosangela.ferreira@ufms.br

${ }^{3}$ Graduada em Nutrição pela Universidade Católica Dom Bosco (UCDB). E-mail: jackeline.minami@hotmail.com 
Resumo: Os hábitos ocidentais caracterizados por padrões alimentares, sedentarismo e estresse psicossocial, associados à predisposição genética, contribuem para o desenvolvimento da síndrome metabólica (SM), estabelecendo impacto deletério no aumento do risco cardiometabólico. O objetivo foi avaliar os fatores de risco para a SM na população nipo-brasileira. Foram estudados 145 indivíduos de ambos os sexos, com idade entre 16 e 79 anos. Observaram-se diferenças entre os sexos em relação à idade, sendo que os valores médios de peso, estatura, CC, PAS e PAD foram maiores nos homens. Entretanto não foram encontradas diferenças quanto ao IMC, IC e glicemia. Constatou-se associação entre sexo e risco de patologias associadas à obesidade, assim como com o estado nutricional e em relação à presença de obesidade I. Identificaram-se adultos não jovens, eutróficos, com IC elevado em ambos os sexos e níveis pressóricos elevados nos homens; com risco muito aumentado de patologias associadas à obesidade em mulheres.

Palavras-chave: síndrome metabólica: doença crônica: estilo de vida.

\begin{abstract}
Western habits characterized by eating patterns, physical inactivity and psychosocial stress associated with genetic predisposition contribute to the development of metabolic syndrome (MS), establishing a deleterious impact on the increased cardiometabolic risk. The objective was to evaluate risk factors for MS in the JapaneseBrazilian population. Have been studied 145 individuals of both sexes, aged between 16 and 79 years. Differences were observed between the sexes in terms of age, with mean values of weight, height, WC, SBP and DBP higher in men. However, there were no differences in $\mathrm{BMI}, \mathrm{IC}$ and blood glucose. It was found association between gender and risk of diseases associated with obesity, as well as nutritional status and for the presence of obesity I. Have been identified not young adults, eutrophic, with IC high in both sexes and high blood pressure in men; with much increased risk of pathologies associated with obesity in women.
\end{abstract}

Key words: metabolic syndrome; chronic disease; lifestyle.

Resumen: Hábitos occidentales se caracterizan por patrones de alimentación, la inactividad física y el estrés psicosocial asociado con la predisposición genética contribuyen al desarrollo del síndrome metabólico (SM), el establecimiento de un impacto nocivo en mayor riesgo cardiometabólico. El objetivo fue evaluar los factores de riesgo para la SM en la población japonesa-brasileña. Se estudiaron 145 sujetos de ambos sexos, con edades comprendidas entre los 16 y 79 años. No se observaron diferencias entre los sexos en relación con la edad, y los valores medios de peso, la altura, CC, PAS y PAD fueron mayores en los hombres. Sin embargo, no hubo diferencias en el IMC, IC y glucosa. Se encontró una asociación entre el sexo y el riesgo de enfermedades asociadas con la obesidad, así como el estado nutricional y la presencia de la obesidad I. No se identificaron los adultos jóvenes, eutróficos, con IC alto contenido de ambos sexos y de la hipertensión arterial en los hombres; con mucho mayor riesgo de patologías asociadas con la obesidad en mujeres.

Palabras clave: síndrome metabólico; enfermedad crónica; el estilo de vida. 


\section{INTRODUÇÃO}

O processo de imigração japonesa no Brasil ocorreu no ano de 1908, em especial no Estado de São Paulo, e atualmente o país conta com a maior comunidade Nikkey residente fora do Japão, com cerca de 1,5 milhões descendentes. Nesse sentido, a imigração okinawana também foi considerada consistente no Estado de Mato Grosso do Sul, sobretudo no ano de 1909, período no qual se deu a construção da Estrada de Ferro Noroeste.

O processo imigratório foi tamanho que influenciou também o atual hábito alimentar dos nipo-brasileiros, sendo este muito semeIhante à dieta ocidental, rica em gorduras saturadas em proteínas e pobre em vitaminas e minerais como o ferro, fato que diverge do padrão alimentar dos japoneses residentes no Japão.

A introdução desses hábitos estabelece um impacto deletério relacionado ao aumento do risco cardiometabólico, fato ocorrido de modo progressivo após a segunda Guerra Mundial, desenvolvendo maior susceptibilidade às doenças crônicas não transmissíveis (DCNT).

O estilo de vida ocidental, como certos padrões alimentares, sedentarismo e estresse psicossocial, associados à predisposição genética, ocorrência do processo de transição nutricional, estabelecido pelo aumento do consumo de alimentos, com alta densidade energética, de produtos proteicos, ricos em gorduras trans e saturadas, dos laticínios gordurosos e diminuição do consumo de frutas, vegetais e cereais, contribuem para o desenvolvimento da síndrome metabólica (SM), definida como um conjunto de fatores de risco que possibilitam o desenvolvimento de doença cardiovascular e diabetes mellitus tipo 2, que por sua vez determinam condições clínicas de doenças crônicas não transmissíveis.

Dessa forma, o objetivo do presente estudo foi avaliar os fatores de risco para a síndrome metabólica na população nipo-brasileira residente na cidade de Campo Grande, Mato Grosso do Sul, Brasil. 


\section{MATERIAL E MÉTODOS}

Trata-se de um estudo epidemiológico, observacional, descritivo, de base populacional. A pesquisa está vinculada a uma ação de extensão da Faculdade de Medicina da Fundação Universidade Federal de Mato Grosso do Sul, intitulada "Dia da Saúde- Avaliação de Síndrome Plurimetabólica e Doenças Infecciosas em Descendentes de Okinawanos" realizada na cidade de Campo Grande, Mato Grosso do Sul, Brasil.

A coleta de dados foi realizada em abril de 2012, sendo analisados 145 indivíduos nipo-brasileiros de ambos os sexos, com idades entre 16 e 79 anos. Os dados foram registrados em um formulário estruturado. Foram excluídos todos os participantes que não preencheram todas as variáveis analisadas.

Realizou-se avaliação nutricional por meio de instrumentos antropométricos como o índice de massa corporal (IMC), utilizando-se a classificação de Lipschitz (1994) para os participantes idosos (magreza $<22 \mathrm{~kg} / \mathrm{m}^{2}$; eutrofia 22 a $27 \mathrm{~kg} / \mathrm{m}^{2}$ e sobrepeso $>27 \mathrm{~kg}$ / $\mathrm{m}^{2}$ ). Para os adultos, a classificação baseou-se em 25 a $29,9 \mathrm{~kg} / \mathrm{m}^{2}$ sobrepesos ou pré-obesos; 30 a $34,925 \mathrm{~kg} / \mathrm{m}^{2}$ obesidade I; 35 a $39,9 \mathrm{~kg} / \mathrm{m}^{2}$ obesidade II; e $\geq 40 \mathrm{~kg} / \mathrm{m}^{2}$ obesidade III (ORGANIZAÇÃO MUNDIAL DA SAÚDE [OMS], 1997). Na aferição do peso corporal e estatura, foram utilizadas balanças profissionais mecânicas (WELMY ${ }^{\circ}$ ) com estadiômetro embutido.

No cálculo do IMC (idoso ou adulto), utilizou-se a expressão matemática abaixo (QUETELET, 1994).

$$
I M C=\frac{\mathrm{P}}{\mathrm{A}^{2}}
$$

Para aferir a circunferência da cintura, utilizaram-se fitas antropométricas metálicas e flexíveis (SANNY ${ }^{\circ}$ ) com definição de medida de 
0,1 cm. Foi classificada de acordo com o sexo e risco aumentado ou muito aumentado de patologias associadas à obesidade. Foram considerados como pontos de corte para o sexo masculino, $\geq 94 \mathrm{~cm}$ risco aumentado e $\geq 102 \mathrm{~cm}$ risco muito aumentado. Para o sexo feminino considerou-se risco aumentado $\geq 80 \mathrm{~cm}$ e risco muito aumentado $\geq$ $88 \mathrm{~cm}$ (OMS, 1998).

Os participantes receberam prospecto sobre orientações nutricionais de alimentação saudável (BRASIL, 2008).

Para o índice de conicidade (IC) foi utilizada medida de peso corporal, estatura e circunferência da cintura, conforme a equação de (VALDEZ, 1999) como um discriminador de risco coronariano elevado.

$$
\text { Índice } C=\frac{\text { Circunferência Cintura }(\mathrm{m})}{\sqrt[0,109]{\frac{\text { Peso Corporal }(\mathrm{kg})}{\text { Estatura }(\mathrm{m})}}}
$$

Foram considerados como pontos de cortes para o sexo feminino 1,18 e para o masculino 1,25, segundo Pitanga e Lessa (2004).

Foi aferida a pressão arterial pelo método auscultatório, com uso do esfigmomanômetro modelo DS44 Tycos da marca Welch Allyn ${ }^{\circledR}$ adequado ao tamanho do manguito à circunferência braquial. A medida foi realizada após cinco minutos de repouso na posição sentada permanentemente imóvel durante esse período. Foram registradas no formulário as pressões sistólica (PAS) e diastólica (PAD) (SOCIEDADE BRASILEIRA DE HIPERTENSÃO [SBH], 2010).

O nível de glicose sanguínea foi mensurado por meio da utilização de aparelho portátil da marca G-tech ${ }^{\circledR}$, através da coleta de uma alíquota de sangue do dedo indicador. Os participantes se encontravam em jejum de 12 horas para a determinação do nível de glicose.

Para o diagnóstico da SM foi utilizada a definição do National Cholesterol Education Programs - Adult Treatment Panel III (NCEP, 
2001), que consiste na presença dos seguintes fatores: obesidade, dislipidemia, hipertensão arterial, resistência à insulina, glicemia de jejum. A determinação do risco cardiovascular associado à SM foi realizada por meio dos valores anormais dos níveis de glicose em jejum ( $\geq 110 \mathrm{mg} / \mathrm{dL}$ ) e pressão arterial ( $\geq 130 />85 \mathrm{mmHg}$ ).

Os dados foram avaliados por meio do programa estatístico GraphPad InStat, adotando nível de 5 \% de significância.

O estudo foi apresentado ao Comitê de Ética e Pesquisa em Seres Humanos da Universidade Federal de Mato Grosso do Sul - Campo Grande (Protocolo n. 2249). Todos os participantes assinaram termo de consentimento concordando em fazer parte do estudo.

\section{RESULTADOS}

A Tabela 1 apresenta os valores médios, desvio padrão e distribuição de frequências das variáveis antropométricas e sociodemográficas pesquisadas. Observaram-se diferenças estatisticamente significativas entre homens e mulheres no que se refere à idade da população estudada $(p=0,02)$. Os valores médios de peso, estatura, $C C$, PAS e PAD foram significativamente maiores no sexo masculino. Entretanto não foram encontradas diferenças significativas quanto aos valores do IMC, IC e glicemia de jejum.

Foi constatada associação entre sexo e risco de patologias associadas à obesidade, sendo a maior frequência representada pelo feminino (64,00 \% vs 39,40 \%). Houve associação entre sexo e estado nutricional $(p=0,02)$. 
Tabela 1 - Média, desvio padrão e percentuais das variáveis analisadas em 145 descendentes okinawanos, 2012, Campo Grande, MS, Brasil

\begin{tabular}{lccc}
\hline \multicolumn{1}{c}{ Características } & $\begin{array}{c}\text { Homens } \\
(\mathbf{n}=\mathbf{6 1})\end{array}$ & $\begin{array}{c}\text { Mulheres } \\
(\mathbf{n}=\mathbf{7 5})\end{array}$ & P ou $\mathbf{x}^{\mathbf{2}}$ \\
\hline Idade (anos) & $47,59 \pm 19,47$ & $52,11 \pm 16,56$ & $0,02^{*}$ \\
Peso (Kg) & $70,36 \pm 16,60$ & $56,98 \pm 8,55$ & $<0,00^{* *}$ \\
Estatura $(\mathrm{m})$ & $1,63 \pm 0,10$ & $1,51 \pm 0,06$ & $<0,00^{*}$ \\
IMC $\left(\mathrm{Kg} / \mathrm{m}^{2}\right)$ & $26,00 \pm 4,70$ & $24,74 \pm 3,32$ & $0,16^{*}$ \\
CC $(\mathrm{cm})$ & $89,56 \pm 12,46$ & $82,62 \pm 10,67$ & $0,00^{*}$ \\
IC & $1,26 \pm 0,11$ & $1,24 \pm 0,13$ & $0,98^{* *}$ \\
PAS (mmHg) & $135,61 \pm 19,75$ & $125,55 \pm 12,73$ & $0,00^{* *}$ \\
PAD (mmHg) & $86,18 \pm 14,85$ & $81,00 \pm 11,69$ & $0,05^{* *}$ \\
Glicemia (mg/dL) & $107,59 \pm 39,04$ & $101,47 \pm 24,13$ & $0,70^{* *}$
\end{tabular}

Risco de patologias associadas à obesidade

Apresenta

Não apresenta
$39,40 \%$

$60,60 \%$
$64,00 \%$

$36,00 \%$
$0,00 * * *$

Classificação risco de patologias associadas à obesidade

Aumentado

Muito aumentado

$62,50 \%$

$37,50 \%$

$45,80 \%$

$54,20 \%$

$0,21 * * *$

Estado nutricional total

$0,02 * * * *$

Magreza

Sim

$6,56 \%$

Não

$93,44 \%$

$12,00 \%$

$88,00 \%$

$0,43 * * * *$

Desnutrição I

Sim

$3,28 \%$

$0,00 \%$

Não

$96,72 \%$

$100,00 \%$

$0,38 * * * *$

Eutrofia

Sim

$45,90 \%$

$54,10 \%$

$54,67 \%$

$45,33 \%$

$0,39 * * * *$

Não

Sobrepeso 


\begin{tabular}{lcrc}
\hline \multicolumn{1}{c}{ Características } & $\begin{array}{c}\text { Homens } \\
(\mathbf{n = 6 1 )}\end{array}$ & $\begin{array}{c}\text { Mulheres } \\
(\mathbf{n}=\mathbf{7 5})\end{array}$ & P ou $\mathbf{x}^{\mathbf{2}}$ \\
\hline Sim & $26,33 \%$ & $30,67 \%$ & $0,70 * * * *$ \\
Não & $73,77 \%$ & $69,33 \%$ & \\
Obesidade I & & & \\
Sim & $14,76 \%$ & $2,67 \%$ & $0,02 * * * *$ \\
Não & $85,24 \%$ & $97,33 \%$ & \\
Obesidade III & & & \\
Sim & $3,29 \%$ & $0,00 \%$ & $0,38 * * * *$ \\
Não & $96,72 \%$ & $100,00 \%$ & \\
\hline
\end{tabular}

* Valores contínuos foram comparados através do teste " $\mathrm{t}$ " de student para amostras independentes paramétricas;

** Valores contínuos foram comparados através do teste Mann-Whitney para amostras independentes não paramétricas;

*** Valores percentuais através do teste Exato de Fisher;

****Valores percentuais através do teste Qui-quadrado;

PAS - pressão arterial sistólica; PAD - pressão arterial distólica; IC - índice de conicidade; IMC- índice de massa corporal; CC- circunferência da cintura; $\chi^{2}$, teste do qui-quadrado

Verificou-se ainda associação estatisticamente significativa entre homens e mulheres no que concerne à presença de obesidade I ( $p=$ $0,02)$, em aproximadamente $3 \%$ da população feminina em relação a $15 \%$ dos indivíduos do sexo masculino.

\section{DISCUSSÃO}

Descendentes de okinawanos que residem no ocidente são considerados mais susceptíveis às patologias que compõe a SM, tais como: diabetes mellitus II, dislipidemia e hipertensão arterial sistêmica. Essa situação decorre das mudanças no estilo de vida e alimentação, a partir da inatividade física e ingestão de dietas ocidentalizadas que possibilitaram o desenvolvimento de alterações no 
metabolismo glicídico (resistência à insulina), aumento do colesterol sanguíneo e frações, aumento da pressão arterial e instalação da obesidade (GOTLIEB, 1990).

Não há consenso quanto aos pontos de corte estabelecidos para caracterização do estado nutricional por antropometria entre outros fatores relacionados à SM (NCEP, 2002; PIMENTA et al., 2013; $\mathrm{SBH}, 2010)$.

Com a finalidade de determinar o estado nutricional dos indivíduos avaliados no presente estudo, alguns parâmetros antropométricos e bioquímicos utilizados são indicadores considerados de grande valia para determinação de SM (PALANIAPPAN et al., 2004).

Neste estudo, a idade encontrada se assemelha ao observado em um estudo realizado no ano de 2013 por Pimenta et al. (2013), que estimaram a probabilidade de agregação e o padrão de combinação de três ou mais componentes da SM em população rural adulta brasileira com idade entre 18 e 60 anos. O mesmo foi observado no estudo de Salaroli (2007), pois também determinaram a prevalência da SM em relação ao sexo e fatores sociais em uma população com idade compreendida entre 25 e 64 anos.

O peso dos indivíduos foi aferido com o objetivo de categorizar o estado nutricional. Observou-se uma média de peso de 70,36 kg entre os homens e 56,98 kg entre as mulheres. O propósito da aferição do peso relaciona-se com o diagnóstico de SM, condição que está diretamente relacionada ao estado nutricional e outros fatores.

Oliveira et al. (2009) avaliaram a prevalência de SM em indivíduos do sexo masculino com idade média de 26,20 anos e observou peso médio de $94,40 \mathrm{~kg}$, que, ao ser comparada com a média de peso apresentada pelos indivíduos do sexo masculino do presente estudo, mostrou-se demasiadamente elevada.

Quanto ao IMC, Correia et al. (2006) encontraram em 128 mulheres, IMC médio de 46,50 kg/m². Rodrigues et al. (2007) en- 
contraram média de $34,75 \mathrm{~kg} / \mathrm{m}^{2}$; valores superiores aos observados nesse estudo, 26,00 e 24,74 kg/m² entre homens e mulheres respectivamente.

Ao aferir a circunferência da cintura, evidenciou-se média de $89,56 \mathrm{~cm}$ em homens e 82,62 cm em mulheres, semelhante ao estudo de Pimenta et al. (2013) que demonstraram 98,5 \% da população masculina com CC < $102 \mathrm{~cm}$ e 78,00\% das mulheres abaixo de $88 \mathrm{~cm}$. O mesmo não evidenciado no estudo de (Rodrigues et al. 2007) que encontrou valores maiores em ambos os gêneros. Portanto, no presente estudo, esses parâmetros não determinaram risco aumentado de patologias associadas à obesidade.

Ao estudar o IC, os valores médios encontraram-se acima da normalidade, 1,26 para o sexo masculino e 1,24 para o feminino o que representa risco coronariano para a população deste estudo. Notou-se divergência com Pitanga e Lessa (2005) e com Vasques et al. (2009) no que se refere ao masculino.

Os resultados vêm demonstrar fortemente a associação entre os níveis pressóricos elevados com SM nessa pesquisa, o que acentua o risco cardiovascular associado para homens, fato corroborado por Rodrigues et al. (2007), e em desacordo com o estudo de Pitanga e Lessa (2005). Quanto à glicemia, segundo NCEP (2002), encontra-se dentro da normalidade para ambos os sexos. MarchiAlves et al. (2012) também não encontraram hiperglicemia no grupo pesquisado.

Houve determinação do risco de doenças associadas à obesidade a partir da avaliação da circunferência da cintura, por constar frequência de 39,40 \% e 64,00 \% entre homens e mulheres respectivamente. Observou-se também a frequência da classificação do risco aumentado associado à obesidade de $62,50 \%$ no sexo masculino e $54,20 \%$ no feminino, com risco muito aumentado. Correia et al. (2006) identificaram risco em mulheres com obesidade mórbida. 
Ao determinar o estado nutricional, observou-se eutrofia em ambos os sexos; fato não encontrado por Fiuza et al. (2008), pois foi evidenciada prevalência de excesso de peso em indivíduos com SM. Isso vem demonstrar que o estado nutricional por antropometria neste estudo não foi determinante como fator de risco para a SM.

\section{CONCLUSÃO}

A caracterização da população estudada identificou adultos não jovens, eutróficos, com índice de conicidade elevado em ambos os sexos e níveis pressóricos elevados no sexo masculino; apresentando risco muito aumentado de patologias associadas à obesidade em muIheres, o que demonstra alterações no estilo de vida em descendentes okinawanos, residentes em Campo Grande, MS.

Cabe aos profissionais de saúde estabelecer ações prospectivas sobre hábitos alimentares saudáveis, prática de exercícios, avaliações nutricionais, no sentido de vislumbrar melhoria da qualidade de vida desses indivíduos.

\section{REFERÊNCIAS}

BRASIL. Guia alimentar para a população brasileira - promovendo a alimentação saudável. Brasília, DF: Ministério da Saúde, 2008.

CORREIA, F. et al. Prevalência da sindrome metabólica: comparação entre os critérios ATPIII e IDF numa população feminina com obesidade severa. Acta Medica Portuguesa, n. 19, p. 289-294, 2006.

FIUZA, M. et al. Metabolic syndrome in Portugal: prevalence and implications for cardiovascular risk-results from the VALSIM Study. Portuguese journal of cardiology : an official journal of the Portuguese Society of Cardiology, v. 27, n. 12, p. 1495--1529, 2008.

GOTLIEB, S. Mortality among Japanese migrants residing in the municipality of São Paulo, Brazil, 1990. Revista de Saúde Pública, São Paulo, v. 24, n. 6, p. 453-467, 1990. 
LIPSCHITZ, D. Screening for nutritional status in the elderly. Primary care, v. 21, n. 1, p. 55-67, mar. 1994.

MARCHI-ALVES, L. M. et al. Componentes da síndrome metabólica na hipertensão arterial. Revista da Escola de Enfermagem da USP, São Paulo, v. 46, n. 6, p. 1348-1353, 2012.

NATIONAL CHOLESTEROL EDUCATION PROGRAMS (NCEP). Third Report of the National Cholesterol Education Program (NCEP) Expert Panel on Detection, Evaluation, and Treatment of High Blood Cholesterol in Adults (Adult Treatment Panel III) final report, v. 106, n. 25, p. 3143-421, dez. 2002.

OLIVEIRA, R. M. S. et al. Influência do estado nutricional pregresso sobre o desenvolvimento da síndrome metabólica em adultos. Arquivos Brasileiros de Cardiologia, v. 92, n. 2, p. 107-112, 2009.

ORGANIZAÇÃO MUNDIAL DA SAÚDE (OMS). Obesidade: impedindo e controlando a epidemia global da obesidade. Relatório Mundial/OMS, 1998.

. Obesidade: impedindo e controlando a epidemia global da obesidade. Relatório Mundial/OMS, p. 3-5, 1997.

PALANIAPPAN, L. et al. Predictors of the Incident Metabolic Syndrome in adults: the insulin resistance atherosclerosis study. Diabetes Care, v. 27, n. 3, p. 788-93, 2004.

PIMENTA, A. M.; FELISBINO-MENDES, M. S.; VELASQUEZ-MELENDEZ, G. Clustering and combining pattern of metabolic syndrome components in a rural Brazilian adult population. São Paulo Medical Journal, v. 131, n. 4, p. 213-9, 2013.

PITANGA, F. J. G.; LESSA, I. Indicadores antropométricos de obesidade como instrumento de triagem para risco coronariano elevado em adultos na cidade de Salvador - Bahia. Arquivos Brasileiros de Cardiologia, São Paulo, v. 85, n. 1, p. 26-31, jul. 2005.

Sensibilidade e especificidade do índice de conicidade como discriminador do risco coronariano de adultos em Salvador, Brasil. Revista Brasileira de Epidemiologia, São Paulo, v. 7, n. 3, p. 259-269, set. 2004.

QUETELET, L. A treatise on man and the development of his faculties. Obesity Research, v. 2, n. 1, p. 72-85, 1994. Disponível em: <http://onlinelibrary.wiley. 
com/doi/10.1002/j.1550-8528.1994.tb00047.x/abstract;jsessionid=F485D0 50B375F600FAA704468D18C835.f03t03>.

RODRIGUES, T. C. et al. Prevalência de síndrome metabólica em uma população de pacientes com excesso de peso (funcionários de um hospital de referência). Revista HCPA, v. 27, n. 10, p. 1-4, 2007.

SALAROLI, L. B. et al. Prevalência de Síndrome Metabólica em estudo de base populacional, Vitória, ES - Brasil. Arquivos Brasileiros de Endocrinologia \& Metabologia, v. 51, n. 7, p. 1143-1152, 2007.

SOCIEDADE BRASILEIRA DE HIPERTENSÃO (SBH). VI Diretrizes Brasileiras de Hipertensão. Arquivos Brasileiros de Cardiologia, São Paulo, v. 95, n. 1, p. 1-51, 2010.

VALDEZ, R. A simple model based index of abdominal adiposity. Journal of Clinical Epidemiology, v. 44, n. 9, p. 955-956, 1999.

VASQUES, A. C. J. et al. Habilidade de indicadores antropométricos e de composição corporal em identificar a resistência à insulina. Arquivos Brasileiros de Endocrinologia \& Metabologia, v. 53, n. 1, p. 72-79, 2009. 
